\title{
Fungal networks and orchid distribution: new insights from above- and below-ground analyses of fungal communities
}

\author{
Lorenzo Pecoraro' ${ }^{1,2}$, Tancredi Caruso4, Lei Cai ${ }^{3}$, Vijai Kumar Gupta ${ }^{5}$, and Zhong-Jian Liu
}

1Shenzhen Key Laboratory for Orchid Conservation and Utilization, The National Orchid Conservation Center of China and The Orchid Conservation and Research Center of Shenzhen, 518114 Shenzhen, China; corresponding author e-mail: lorenzo.pecoraro@gmail.com ${ }^{2}$ Center for Biotechnology \& BioMedicine and Division of Life \& Health Sciences, Graduate School at Shenzhen, Tsinghua University, 518055 Shenzhen, China

${ }^{3}$ State Key Laboratory of Mycology, Institute of Microbiology, Chinese Academy of Sciences, 100101 Beijing, China

${ }^{4}$ School of Biological Sciences, Queen's University of Belfast, BT9 7BL Belfast, Northern Ireland

${ }^{5}$ Department of Chemistry and Biotechnology, ERA Chair of Green Chemistry, School of Science, Tallinn University of Technology, 12618 Tallinn, Estonia

Abstract: Orchids are critically dependent on fungi for seedling establishment and growth, so the distribution and diversity of orchids might depend on the associated fungal communities. We characterised the communities associated with eight orchid species in three Mediterranean protected areas, using a combination of above-ground analyses of sporophores and below-ground molecular analyses of orchid root samples. In three years of sporophore collection in 25 plots around flowering orchid plants, 268 macrofungal species belonging to 84 genera were observed. Statistical analyses indicated a correlation between macrofungal diversity and orchid community variation, regardless of the effect of environmental and spatial factors characterizing the investigated orchid sites. Fungal ITS-DNA PCR amplification, cloning, and sequencing revealed Rhizoctonia-like fungi belonging to Ceratobasidiaceae (26\%), Tulasnellaceae (22.5 $\%)$, and Sebacinaceae (3.5\%), as well as other basidiomycetes and ascomycetes, in the roots of 99 orchid plants. Mycorrhizal specificity was low but co-occurring orchid species showed preferences for different partners. The diverse macrofungal communities found in the sites may contribute to orchid community variation without colonizing the orchid roots. Molecular analyses revealed a segregation of associated fungi, which may contribute to Mediterranean orchid coexistence in nature.

\author{
Key words: \\ endophytic fungi \\ macrofungal communities \\ Mediterranean \\ molecular ecology \\ mycorrhizas \\ orchid-fungus relationships \\ spatial distribution \\ species coexistence
}

Article info: Submitted: 7 August 2017; Accepted: 27 January 2018; Published: 12 February 2018.

\section{INTRODUCTION}

The family Orchidaceae is particularly dependent on fungal symbionts: orchids have obligate associations with mycorrhizal fungi, because of their initially mycoheterotrophic life-style (Leake 1994). Orchid species rely on their fungal associates for the provision of nutrients, at least during the non-photosynthetic protocorm stage of development, and in some cases throughout their life (Rasmussen 1995). Because orchids are critically dependent on fungi for seedling establishment and growth, the distribution and diversity of orchids might be expected to be correlated with the composition of the associated fungal communities. The relationship between orchid distribution, abundance, and mycorrhizal diversity has been analysed at different spatial scales (McCormick et al. 2009, Jacquemyn et al. 2012). Davis et al. (2015) showed that the specialized association with Sebacina did not limit the geographical range of the widely distributed Australian orchid Pheladenia deformis. Jacquemyn et al. (2014), however, demonstrated that mycorrhizal fungi could mediate terrestrial orchid species coexistence, driving niche partitioning. The extent to which fungal communities may determine the distribution and dynamics of orchid populations remains unclear (McCormick et al. 2012, McCormick \& Jacquemyn 2014).

Nearly all previous studies carried out to determine fungal diversity in orchid sites have been based on experimental seed-sowing and/or sequencing of fungal DNA from orchid tissues (Dearnaley 2007, McCormick \& Jacquemyn 2014). Such experiments are limited in only providing information on the fungi that effectively establish associations with the orchids (Těšitelová et al. 2012). The presence and distribution of potentially symbiotic fungi, as well as the diversity of the associated fungal community in nature have remained unexplored (Oja et al. 2015).

Orchid distribution could be influenced not only by the presence and abundance of suitable mycorrhizal fungi, but also by the total diversity of associated fungi naturally occurring in the field. There is a possibility of various combinations of direct and indirect interactions between and within the fungi, and also

\section{๑) 2018 International Mycological Association}

You are free to share - to copy, distribute and transmit the work, under the following conditions:

Attribution: $\quad$ You must attribute the work in the manner specified by the author or licensor (but not in any way that suggests that they endorse you or your use of the work).

Non-commercial: $\quad$ You may not use this work for commercial purposes.

No derivative works: You may not alter, transform, or build upon this work.

For any reuse or distribution, you must make clear to others the license terms of this work, which can be found at http://creativecommons.org/licenses/by-nc-nd/3.0/legalcode. Any of the above conditions can be waived if you get permission from the copyright holder. Nothing in this license impairs or restricts the author's moral rights. 
between the plants and the fungi. Fungi forming associations with non-orchid plants may indirectly affect orchid diversity through contributing to the structure of plant communities, a major factor influencing the establishment and growth of orchid species (Kosaka et al. 2014).

In this study, we investigated abiotic and biotic factors influencing both putative fully photosynthetic and partially mycoheterotrophic orchid diversity and distribution in three Mediterranean protected areas, across forest and meadow habitats. We characterized the fungal communities associated with eight orchid species (six fully-photosynthetic meadow and two partially mycoheterotrophic forest species) in calcareous mountain sites using a combination of above- and below-ground analyses. We aimed to test the hypothesis that variation in above-ground fungal community structure, as reflected in the sporophores produced, is a predictor of the orchid species assemblage. We tested this hypothesis by eliminating possible effects of other major covariates quantifying the environmental and geographical characteristics of the sites, including vegetation types (e.g. shrub and tree cover vs. meadows). We also reversed the predictors and responses used to test the hypothesis that variation in the orchid assemblage was a predictor of the above-ground fungal community structure. Further, we also examined associated below-ground fungal taxa using molecular tools to determine whether orchid species identity was a predictor of the associated root fungal community.

\section{MATERIAL AND METHODS}

\section{Study sites and species}

The study was conducted in three geographically distinct protected areas in central Italy, the 'Monte Cetona', 'Monte Penna' (specifically on 'Monte Rotondo'), and 'Cornate di Gerfalco' Nature Reserves. The areas consist of calcareous mountains characterized by Mediterranean montane dry grasslands with Bromus erectus dominant among herbaceous plants. These meadow habitats are surrounded by forests with various broad-leaved trees including species of Acer, Fagus sylvatica, Fraxinus ornus, Ostrya carpinifolia, and Quercus cerris, mixed with conifers such as Pinus nigra. All the sites are located on the top of the three mountains, at 755-1047 m a.s.l.

In spring 2006, within each site, plots of $20 \mathrm{~m}$ diam were established in open meadow and surrounding forest habitats based on the presence of fully-photosynthetic and partially mycoheterotrophic orchid species respectively. Eight orchid species were found: six meadow orchids (Anacamptis morio, Himantoglssum adriaticum, Ophrys bertolonii, Orchis pauciflora, O. provincialis, and O. tridentata), and two forest orchids (Cephalanthera damasonium, and C. longifolia). Each plot was randomly established around a flowering orchid of one of the eight species in the investigated sites, resulting in 25 plots. The centre of each plot was mapped using highprecision GPS (Trimble Navigation, Sunnyvale, CA) and marked with a metal stake. In order to assess environmental and geographical conditions of the study plots, we recorded the: habitat (meadow or forest), \% vegetation cover (divided into tree, shrub, herb, and lichen-moss percentages), litter cover \% (ground surface covered by plant debris), rock cover \% (coherent material), stone cover \% (non-coherent material), micro-topography (scored 1-3 related to increasing irregularity of the ground surface), elevation above sea level, slope \%, and orientation (Table S1 Supporting Information).

\section{Above-ground analysis of fungal communities}

Field surveys for above-ground macrofungal sporophores more than $1 \mathrm{~mm}$ diam (Arnolds 1981), were carried out every week during the main basidiomata and ascomata production seasons, essentially autumn (September-December) and spring (April-June) respectively (when rainfall occurs with suitable temperatures), from September 2006 to June 2009. Sporophores were collected and identified by macroand microscopic observations of fresh material. Notes on morphological characters and ecological conditions were recorded in the field, and samples were taken to the laboratory for microscopic examination. Numbers of sporophores within plots were recorded during every survey visit for each species found. Material was dried and voucher specimens deposited in the Herbarium Universitatis Senensis (SIENA).

Monitoring was for three consecutive years to provide a reasonable representation of the fungal communities present (Vogt et al. 1992). The structure of the macrofungal communities within each plot was expressed by assembling all the data on species identity and productivity.

\section{Below-ground analyses of orchid root fungal associates}

\section{Orchid sample collection}

In early summer of 2007 and 2008, during the flowering period, roots were collected from 11-15 adult individuals of the eight orchid species, resulting in 99 samples from the three study areas (Table S2 Supporting Information). Roots were rinsed in water and carefully scrubbed with a brush to remove most soil debris. They were then treated in an ultrasonic bath (three cycles of $30 \mathrm{~s}$ each) to remove any remaining soil particles and to minimize the risk of detecting all microscopic organisms adhering to the root surface. Thin cross sections of fresh root samples were checked for mycorrhizal colonization by light microscopy. Root fragments exhibiting high fungal colonization were immediately processed for fungal isolation. Root portions to be used for molecular analysis were frozen in liquid nitrogen and stored at $-80^{\circ} \mathrm{C}$.

\section{Fungal isolation}

Five or six roots per plant were surface-sterilized with consecutive washes of $1: 5$ sodium hypochlorite (30 s) and three rinses of sterile water. Ten 3-5 mm long pieces from each root were cultured on malt extract agar (MEA) and potato dextrose agar (PDA) amended with gentamycin (40 mg/l) and/ or chloramphenicol $(50 \mu \mathrm{g} / \mathrm{ml})$. Petri dishes were incubated at room temperature $\left(20-25^{\circ} \mathrm{C}\right)$ in the dark for up to two months to allow the development of slow-growing mycelia.

DNA extraction, PCR amplification of fungal ITS, cloning, and sequencing

Both total DNA from orchid root samples and DNA from isolated fungi were extracted using the cetyltrimethyl ammonium bromide (CTAB) procedure modified from Doyle 
\& Doyle (1990). Fungal ITS regions were PCR-amplified using the primer pair ITS1F/ITS4 (Gardes \& Bruns 1993) following the methods described in Pecoraro et al. (2017) for PCR reaction, thermal cycling, purification and cloning of PCR products. Cloned ITS inserts were purified with a Plasmid Purification Kit (Qiagen) and sequenced with the same primer pair used for amplification. Dye sequencing was carried out on ABI 310 DNA Sequencer (Applied Biosystems, Carlsbad, CA).

\section{Sequence analysis}

Sequences were edited to remove vector sequences and to ensure correct orientation and assembled using Sequencher 4.1 for MacOsX (Genes Codes, Ann Arbor, MI). Sequence analysis was conducted with BLAST searches against the National Center for Biotechnology Information (NCBI) sequence database (GenBank; http://www.ncbi.nlm.nih. gov/BLAST/index.html) to determine the closest sequence matches that enabled taxonomic identification. The fungal community associated with the roots of the investigated species has been partly described in Pecoraro et al. (2012a, b, 2013, 2015, 2017). Molecular analyses were performed for 28 additional orchid individuals from two different species A. morio (15 samples) and 0 . provincialis (13 samples). Fungal DNA sequences amplified from the eight analysed species are deposited in GenBank (Accession nos: A. morio KX461964-KX461987, C. damasonium KT122776-KT122789, C. longifolia KT122767-KT122775, H. adriaticum JQ685234JQ685249, O. bertolonii KP868521-KP868544, O. pauciflora JQ723318-JQ723347, O. provincialis KX461988- KX462007, and O. tridentata JN683860-JN683859).

Phylogenetic analysis was conducted with Mega v. 5.0 (Tamura et al. 2011). Sequences were aligned with Clustal $X$ v. 2.0 (Larkin et al. 2007) and neighbour-joining trees against selected database sequences were constructed using Kimura 2-parameter distances, with bootstrapping of 1000 replicates (Felsenstein 1985). Due to the phylogenetic distance between the fungi identified from the roots of investigated orchids, distinct phylogenetic analyses were performed. The globose and ellipsoid-spored Tulasnella eichleriana and T. tomaculum were used as outgroups to root the tulasnelloid fungal tree, while the ceratobasidioid fungi tree was rooted with Laccaria bicolor and Tricholoma portentosum.

\section{Statistical analyses}

We performed a Principal Component Analysis (PCA) on the correlation matrix of environmental variables (habitat, tree, shrub, herb, and lichen-moss cover $\%$, litter cover $\%$, rock cover $\%$, stone cover $\%$, micro-topography, elevation above sea level, slope \%, and orientation; Legendre \& Legendre 1998, Gotelli \& Ellison 2004). We then used the axes from this PCA to define major environmental gradients that potentially correlated with species distribution (environmental correlates). Using PCA axes as proxies for environmental gradients allowed us to remove the issue of collinearity of predictors (Gotelli \& Ellison 2004) in further multivariate analysis of fungi and orchid community structure. Spatial information (UTME coordinates) was used to apply PCA to neighbour joining matrices, and so described possible spatial dependence of plots at multiple scales (PCNM; Borcard \&
Legendre 2002). The method produces several vectors that reflect possible spatial structure in the data, and the subset of vectors eventually used in our analysis was selected by a multivariate extension of the Akaike information criterion (AIC; Dray et al. 2006). We consequently created a set of environmental and spatial vectors or factors potentially accounting for variance in the community structure of fungi and orchids. Given the observational nature of this study, multivariate patterns in above-ground fungi (sporophores) and orchids can reciprocally be used as predictors of each other, but results might change depending on the spatial structure of each community, which might not be the same at all scales. In one set of analyses we used orchids as predictors of fungi, and in another we swapped predictors for responses. For fungi producing sporophores aboveground, we had information on relative abundance, while for the orchids there was only presence/absence information. When we used fungi in response to orchids, we Hellingertransformed raw data prior to apply distance based RDA (dbRDA) to ensure no inflation of the weights accorded to rare species and omit joint absences (Legendre \& Gallagher 2001). We used partial-dbRDA to test for any unique effects of orchids by controlling for covariation between orchids on the one hand, and the sum of environmental correlates and spatial autocorrelation on the other. To use orchids as a predictor of fungi, we calculated a PCoA of the orchid Jaccard matrix and extracted major PCoA axes to measure orchid community structure. These axes were then used as predictors (i.e. explanatory axes) of the fungal communities. Given the number of available plots, we had to limit the number of explanatory PCoA axes to a maximum of three to reduce the issue of over-fitting to acceptable levels (i.e. low predictors to observations ratio). The same overall procedure was used to test for the effect of fungi on orchids, and we used a permutational approach to test for the statistical significance of effects (Oksanen et al. 2012).

We also performed a similar multivariate analysis on the fungal community molecularly detected in the roots to test whether different fungal assemblages were associated with different orchids. We calculated environmental and spatial factors using the same methods applied to the orchid and above-ground fungal datasets and tested for any effect of orchid species on the fungi after excluding environmental and spatial factors. All analyses were performed in R 3.1 (R Core Team 2015).

\section{RESULTS}

\section{Macromycete diversity and community structure in orchid sites}

In total, 6365 macromycete sporophores were recorded, representing 268 species in 84 genera (Table S1 Supporting Information); 157 species in 59 genera were harvested in the meadow plots, and 153 species in 62 genera in the forest plots. Forty-two taxa were found in both habitats (Table S1 Supporting Information).

Several species-rich saprobic genera, such as Marasmius and Mycena, were observed. Three of nine identified Marasmius species (M. epiphylloides, $M$. epiphyllus, and 
M. quercophilus) yielded 437, 688, and 545 basidiomes respectively, whereas Mycena, represented by 35 taxa, including $M$. clavicularis (600 basidiomata), showed the highest species richness of the whole community (Table S1 Supporting Information).

The mycorrhizal fungal community was represented by 63 species in 12 genera of basidiomycetes, out of 1127 basidiomes collected. Amongst these, Inocybe species accounted for $27.5 \%$ of the basidiomes and $20.6 \%$ of the taxonomic diversity (13 species), while Russula (10 identified species), was the second species-richest group. Suillus granulatus dominated basidiome production amongst the ectomycorrhizal fungi, with 153 basidiomes recorded, and the second highest was $I$. splendens with 108 basidiomes collected in the forest plots of 'Monte Penna' (Table S1 Supporting Information). Of all taxa identified, 42 species were recorded in two out of three of the studied areas, while only 20 were collected in all three investigated Nature Reserves. Eight of these 20 taxa belong to Mycena, with M. abramsii, M. aetites, M. flavoalba, M. pura, M. pura f. alba, M. rosea, and M. vitilis collected both in meadow and forest habitats, whereas $M$. leptocephala was only found in meadow plots. From the remaining taxa recorded in all the three studied areas, Conocybe tenera, Crinipellis scabellus, Lepiota clypeolaria, and Panaeolus acuminatus were only present in meadow plots; Hygrophorus eburneus, Lepista nuda, and Tricholoma album were exclusively associated with the forest habitat; and Clitocybe costata, C. semiglobata, $H$. sinapizans, and T. atrosquamosum were found in both meadow and forest plots.

Microscopical observation showed that cortical cells of Acamptis morio and Orchis provincialis roots were densely colonized by fungal hyphae forming typical orchid mycorrhizal intracellular coils (pelotons), thus confirming the high level of fungal colonization previously described in these orchid species (Pecoraro et al. 2012a, b, 2013, 2015). Rhizoctonialike hyphae emanating from pelotons were observed in the majority of root samples.

Endophytic fungi exhibiting Rhizoctonia-like morphological features were obtained by in vitro isolation from the roots. Molecular identification of these isolates revealed that they belonged to Ceratobasidiaceae (Table S3 Supporting Information). A non-ceratobasidioid basidiomycete was also isolated from $A$. morio sample MC4, the closest match for the sequence amplified from the culture being Schizophyllum commune (Schizophyllaceae, Table S3 Supporting Information).

Direct root DNA amplification mostly yielded fungal ITS sequences showing similarity to Rhizoctonia fungi belonging to Ceratobasidiaceae (56\% sequences) and Tulasnellaceae (11\% sequences). A variety of ascomycetes was also identified in 7 out of $14 \mathrm{~A}$. morio, and 4 out of 13 O. provincialis individuals (Table S3 Supporting Information).

PCR products were obtained with the universal fungal primer pair ITS1F/ITS4 from 88 out of 99 analysed samples from the eight orchid species. More than half of the samples (52\%) yielded Rhizoctonia sequences with an identity in Ceratobasidiaceae (26\%), Tulasnellaceae (22.5\%), and Sebacinaceae (3.5\%), whereas $33 \%$ of orchids were only colonized by ascomycetous fungi, notably Exophiala sp., Leohumicola sp.,

Fig. 1. Neighbour-joining phylogenetic tree showing the relationship between the Ceratobasidiaceae sequences obtained from the analysed orchid species $\left({ }^{*}\right)$ and selected database relatives. Kimura 2-parameter distances were used. Bootstrap values are based on percentages of 1000 replicates. The tree was rooted with Laccaria bicolor and Tricholoma portentosum as outgroups.

\section{Orchid root fungal diversity and mycorrhizal specificity}

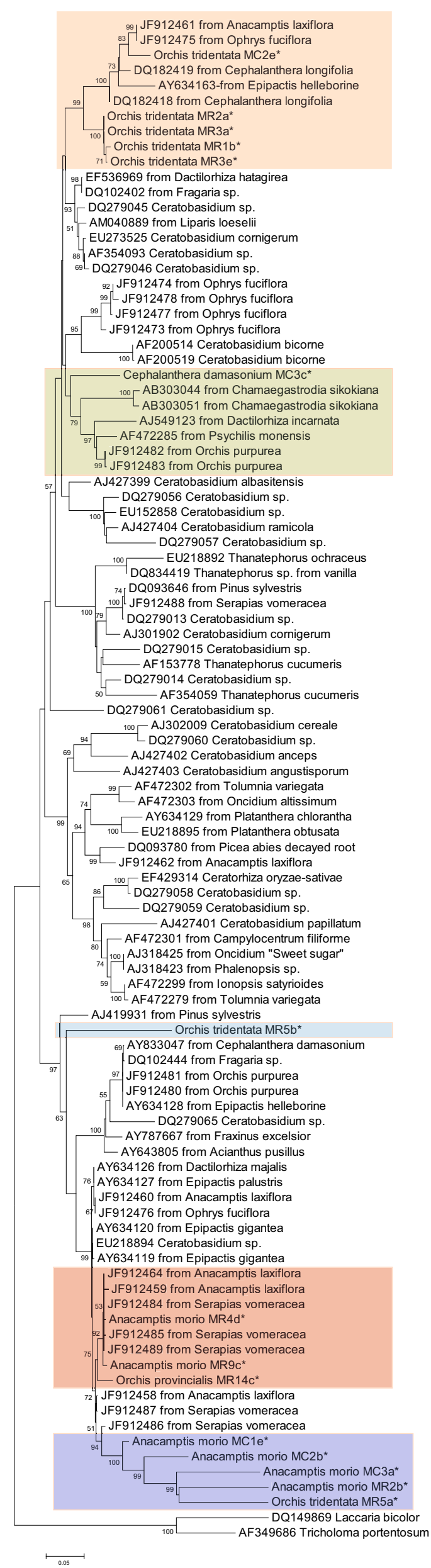


58- DQ925564 from Cypripedium calceolus

68. DQ925552 from Cypripedium macranthos

68 DQ925554 from Cypripedium calceolus

DQ25555 from Cypripedium parviflorum

DQ925557 from Cypripedium parviflorum

${ }_{99}$ JP926492 from Orchis purpurea

${ }_{98}\left[\begin{array}{l}\text { JF926492 from Orchis purpurea } \\ \text { JF926491 from Orchis purpurea }\end{array}\right.$

${ }_{100}$ JF926489 from Orchis purpurea

JF926487 from Orchis purpurea

JF926484 from Orchis purpurea

JF926488 from Orchis purpurea

JF926485 from Orchis purpurea

JF926490 from Orchis purpurea

DQ925572 from Cypripedium montanum

Himantoglossum adriaticum CG2a

100 - Himantoglossum adriaticum CG7b

Himantoglossum adriaticum CG7 $\mathrm{e}^{*}$

- Himantoglossum adriaticum CG5a*

Himantoglossum adriaticum CG5a

Himantoglossum adriaticum CG1a*

EU583691 from Orchis $x$ bergonii

Himantoglossum adriaticum MC2a

Himantoglossum adriaticum MC3b

Himantoglossum adriaticum MC $3 \mathrm{e}^{*}$

- DQ925576 from Cypripedium montanum

DQ925599 from Cypripedium arietinum

DQ925600 from Cypripedium arietinum

DQ925600 from Cypripedium arietinum

JF926481 from Ophrys fuciflora

Ophrys bertolonii $7 \mathrm{~b}^{*}$

Ophrys bertolonii $7 \mathrm{a}^{*}$

Ophrys bertolonii $6 b^{*}$

Ophrys bertolonii $6{ }^{*}$

Orchis provincialis MR18b

Orchis provincialis MR21b

Orchis provincialis MR18d

2. Orchis provincialis MR22b

${ }_{74}$ Orchis provincialis MR21a*

Orchis provincialis MR22a

Orchis provincialis MR22

O 52533 from Cypriped

DQ925633 from Cypripedium

DQ925633 from Cypripedium guttatum

DQ925635 from Cypripedium guttatum

96 DQ925624 from Cypripedium montanum

DQ925629 from Cypripedium fasciculatum

DQ925615 from Orchis mascula

- Orchis pauciflora $4 \mathrm{CGe}^{*}$

Orchis pauciflora $4 \mathrm{CGC}^{*}$

${ }_{83} \mathrm{~L}$ Orchis pauciflora $4 \mathrm{CGa}^{*}$

50. DQ925597 from Cypripedium regina

DQ925593 from Cypripedium reginae

DQ925595 from Cypripedium regina

JF926472 from Ophrys fuciflora

JF926476 from Ophrys fuciflora

JF926479 from Ophrys fuciflo

- JF926479 from Ophrys fuciflora

JFF26475 from Ophrys fuciflora

JF926473 from Ophrys fuciflora

JF926478 from Ophrys fuciflora

JF926474 from Ophrys fuciflora

JF926471 from Bromus erectus

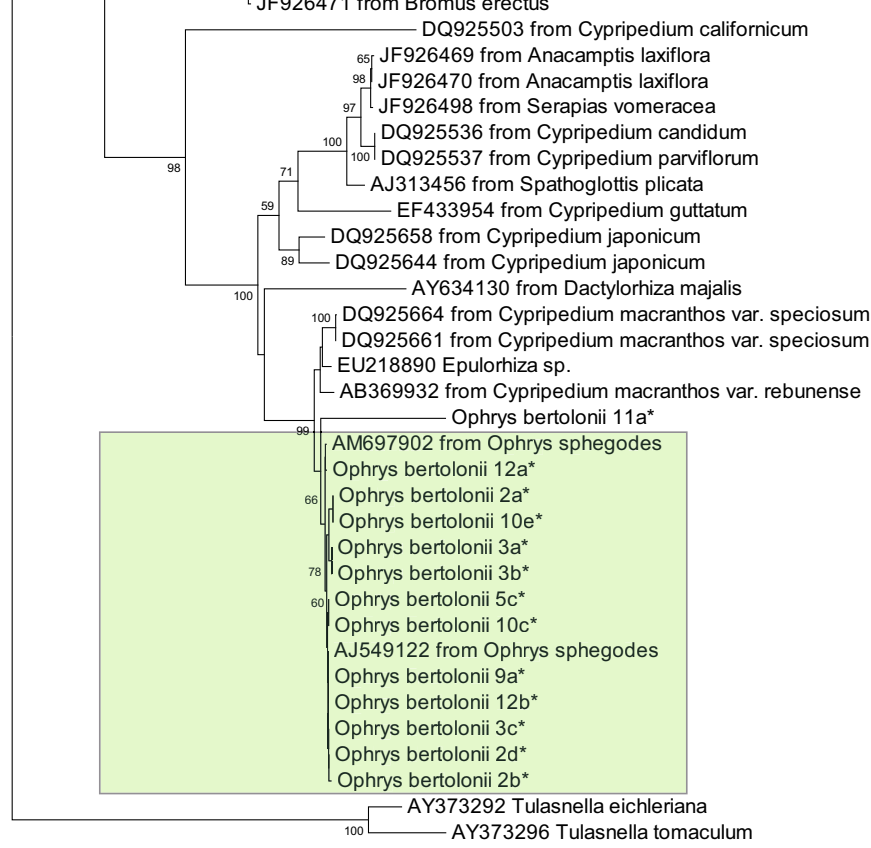

JF02640 from An Cypripedium calf

JF926470 from Anacamptis laxiflora

JF926498 from Serapias vomerace

DQ925536 from Cypripedium candidum

AJ313456 from

DQ925664 from Cypripedium macranthos var. speciosum

DQ925661 from Cypripedium macranthos var. speciosum

phrys bertolonii $2 a^{*}$

AJ549122 from Ophrys sphegodes

Ophrys bertolonii $9 a^{*}$

phrys bertolonii 12

phrys bertolonii $2 a^{*}$

AY373292 Tulasnella eichleriana

AY373296 Tulasnella tomaculum
Leptodontidium sp., Tetracladium sp., and Fusarium sp., and $9 \%$ by non-Rhizoctonia-like basidiomycetous endophytes, including Cryptococcus carnescens, Hymenogastraceae, and Tomentella species. In 13 orchid samples, both Rhizoctonia and non-Rhizoctonia fungal sequences were amplified (Table S2 Supporting Information).

Phylogenetic analysis of the Rhizoctonia-like associates revealed relationships within Ceratobasidiaceae and Tulasnellaceae (Figs 1-2). Sequences amplified from roots and from isolated fungi could be aligned with GenBank sequences from fungal strains and sporophores, as well as from several plants, including orchid and nonorchid species. In the neighbour-joining tree from the Ceratobasidiaceae dataset (Fig. 1), sequences from the orchids segregated into five main clusters. In particular, most sequences from $O$. tridentata co-segregate in a cluster including GenBank fungal sequences from different fully photosynthetic meadow orchids (e.g. A. laxiflora and $O$. fuciflora), and partially mycoheterotrophic orchids growing in open forest habitats (including $C$. longifolia and Epipactis helleborine). An O. tridentata sequence from MR5 (clone b) segregated into a distinct cluster with ones from orchids of the same (O. purpurea) and different genera (Acianthus pusillus, $C$. damasonium, and $E$. helleborine), as well as sequences from non-orchid plant species (Fragaria sp. and Fraxinus excelsior) and from a Ceratobasidium isolate (DQ279065). The remaining $O$. tridentata sequence (MR5, clone a) clustered with the majority of $A$. morio sequences in our study, whereas $A$. morio sequences from MR4d and MR9c fell in one including an O. provincialis sequence from MR14c and GenBank sequences from the green meadow orchids $A$. laxiflora and $S$. vomeracea. The only Ceratobasidium sequence we amplified was from Cephalanthera damasonium and clustered with ones from the achlorophyllous forest orchid Chamaegastrodia sikokiana, the photosynthetic meadow orchids Dactylorhiza incarnata and O. purpurea, and the epiphytic orchid species Psychilis monensis.

The phylogenetic tree of Tulasnellaceae showed the investigated orchids associated with different groups of tulasnelloid fungi (Fig. 2). Tulasnellaceae sequences obtained from $H$. adriaticum likely represented two or three distinct taxa. Ones from $O$. bertolonii segregated into two clusters, the smaller including GenBank sequences amplified from Cypripedium arietinum and $O$. fuciflora, and the larger including ones from $O$. sphegodes. On the basis of ITS sequences, fungi detected in 0 . pauciflora and $O$. provincialis spanned a smaller range of variation, appearing to belong to a single taxon for each orchid species, the two orchid taxa being represented by closely related fungi.

Fig. 2. Neighbour-joining phylogenetic tree showing the relationship between the Tulasnellaceae sequences obtained from the analysed orchid species $\left(^{*}\right)$ and selected database relatives. Kimura 2-parameter distances were used. Bootstrap values are based on percentages of 1000 replicates. The tree was rooted with Tulasnella eichleriana and T. tomaculum as outgroups. 
Table 1. Variance decomposition of fungi (sporophores) community structure. The effect of orchids was quantified and tested by controlling for the covariation ("Conditioned") due to measured environmental variables (environment) and geographical position of plots (space).

\begin{tabular}{lll}
\hline Source & Variance (\%) & P-value \\
\hline \hline Conditioned (environment + space) & 49 & \\
Orchid effect & 7 & $>0.05$ \\
Residual & 44 & \\
\hline
\end{tabular}

\section{Statistical results}

We found variation in tree, shrub, and litter cover was negatively correlated with variables such as slope, herbaceous, lichen/moss, rock and stone cover. This accounted for $57 \%$ of the environmental variation between plots (Fig. S1a Supporting Information, PC1) and is due to including meadows and woodlands in the sampling design. Second was an elevation gradient (PC2, $18 \%$ ), with meadow and woodland plots at various altitudes. These first two PCA axes were therefore used to define: (1) a vegetation index (PC1) that distinguished meadows from woodlands (S1b Supporting Information) but also accounted for variation in the measured variables within meadows and woodlands; and (2) an elevation gradient index (PC2).

Spatial, environmental, and orchid factors could account for $56 \%$ of the total variance in macrofungi (Table 1 ). The sum of environmental and spatial variation represented the largest fraction ( $49 \%$ ) while the unique effect of orchids (7\%) was not statistically significant at $\mathrm{p} 0.05$ after removing environmental and spatial variation. A similar scenario was observed when we reversed predictors and response variables, but orchids
Table 2. Variance decomposition of orchids community structure. The effect of fungi (sporophores) was quantified and tested by controlling for the covariation ("Conditioned") due to measured environmental variables (environment) and geographical position of plots (space).

\begin{tabular}{lll}
\hline Source & Variance (\%) & P-value \\
\hline \hline Conditioned (environment + space) & 70 & \\
Fungi effect & 7 & $<0.05$ \\
Residual & 22 & \\
\hline
\end{tabular}

were strikingly more structured in terms of environmental and spatial variation, which accounted for $70 \%$ of total variance (Table 2). However, while fungi accounted just for $7 \%$ of orchid variation, this effect was still significant after removing environmental and spatial variation. Unconstrained PCoA ordination of fungi (Fig. 3) and orchids (Fig. 4) illustrates some key aspects of these results. The fungal community was less structured than the orchid community, the first two PCoA axes of the fungal communities accounting for $16 \%$ and $13 \%$ of total variance respectively; something to be partly expected given the high dimensionality (i.e. very high species richness relative to the number of sampled plots) of the fungal community. Instead, in the case of orchids, they respectively accounted for $41 \%$ and $24 \%$ of total variance. Also, the two communities were structured in a different way: in the case of orchids the most important gradient (PCoA 1, $41 \%$ ) was environmental and basically due to the difference between meadow and woodland plots (Fig. 4) by construction, while the second gradient (PCoA 2, $24 \%$ ) separated one site ('Monte Penna') from the other two ('Monte Cetona' and 'Cornate di Gerfalco'). Instead, in the case of fungi the

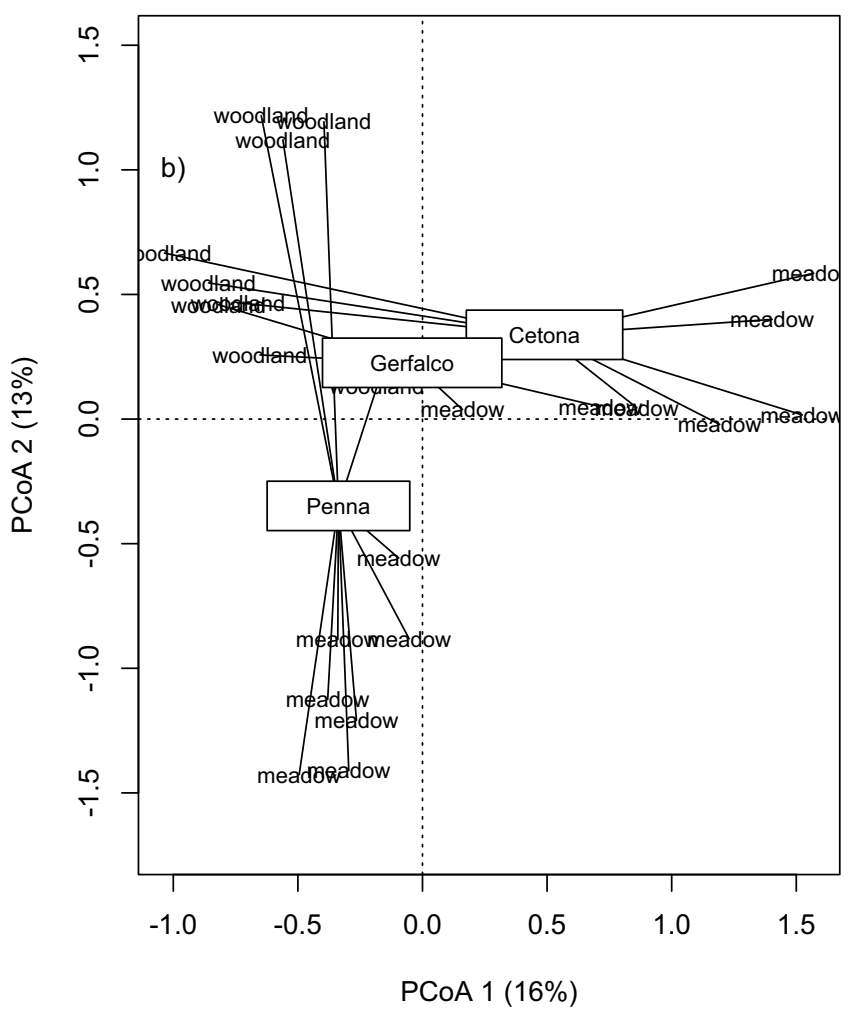

Fig. 3. PCoA ordination of the fungal community (sporophores) after Hellinger transformation. The two figure panels show exactly the same data but with different labels. Left, plots labelled by site and clustered habitat (meadow vs woodland); Right, plots labelled by habitat and clustered by site (Penna, Gerfalco and Cetona). 

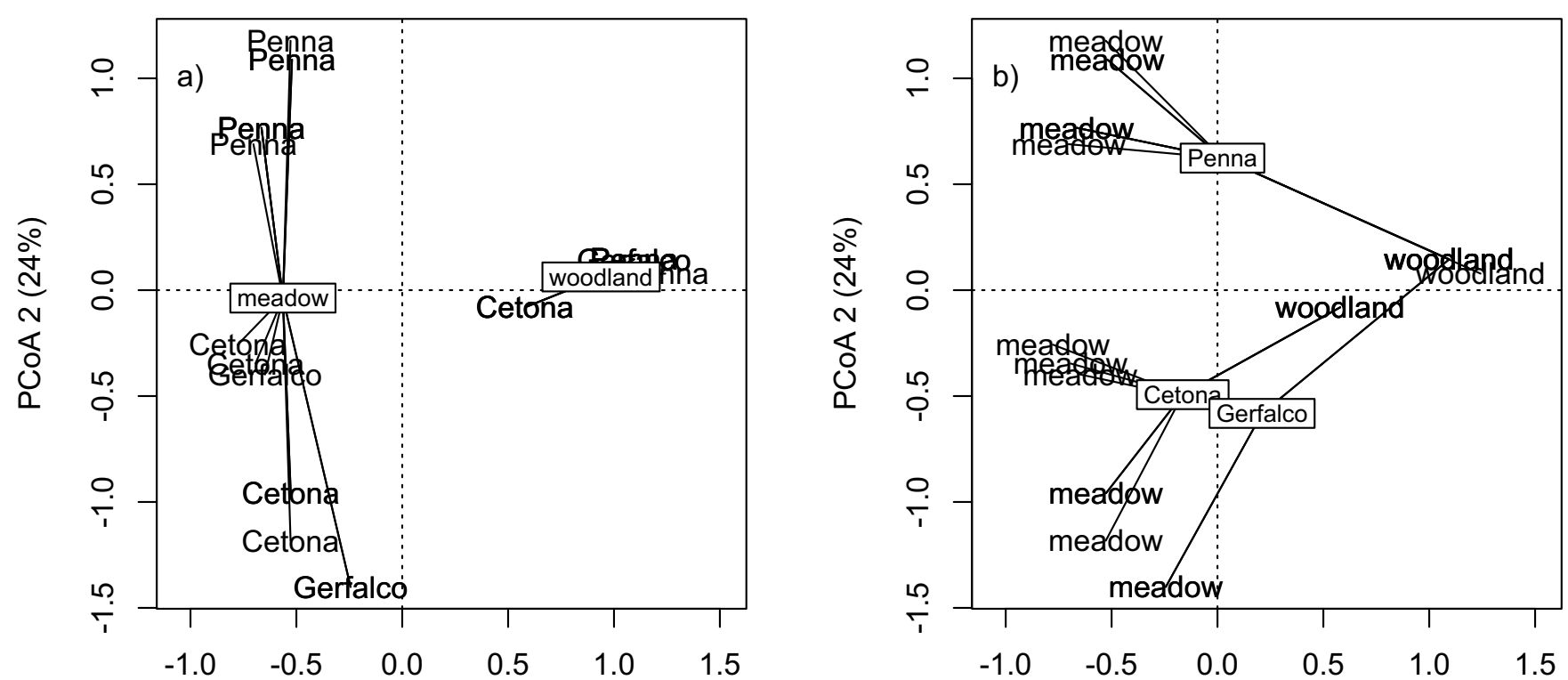

Fig. 4. $\mathrm{PCoA}$ ordination of the jaccard distance matrix of the orchid community. The two figure panels show exactly the same data but with different labels. Left, plots labelled by site and clustered habitat (meadow vs woodland); Right, plots labelled by habitat and clustered by site (Penna, Gerfalco and Cetona).

first gradient was in terms of site separation and the second gradient in the habitat separation, but the clustering pattern was much more confused and in any case the variance of the first two axes was similar and low.

We also performed a similar multivariate analysis on the fungal assemblages molecularly detected in the roots of the different species of orchids. We calculated environmental and spatial factors using the same methods applied to the orchid and above ground fungal datasets, by keeping a record of the plot from which each sequenced orchid root was collected. Environmental and spatial factors together accounted for a very small $(<1 \%)$ fraction of root fungal community variation. After removing this effect, orchid species identity significantly affected the root fungal composition, accounting for about $15 \%$ of total community variance in the root. Environmental data, spatial position of plots and orchid species identity could account for $31 \%$ of total variance in the fungal assemblage detected in roots: $19 \%$ was due to the sum of environmental plus spatial effects, and $13 \%$ to the effect of orchid species identity. This last fraction was statistically significant at $p<0.05$. A PCoA ordination clarifies the relationship between orchid species (Fig. 5), with $H$. adriaticum, $\mathrm{O}$. bertolonii, and $\mathrm{O}$. pauciflora forming one cluster, and all the other species another.

\section{DISCUSSION}

\section{Mediterranean orchid diversity and distribu- tion can be influenced by macromycetes com- munities}

The investigated orchid species shared their meadow and forest habitats with a wide range of basidiomycetous and ascomycetous fungi, with 268 macrofungal species identified based on sporophore characters. Several macrofungal species colonizing the studied plots are known as orchid mycorrhizal fungi. Among the 63 mycorrhizal basidiomycetous taxa developing basidiomes in the sampled plots, were 13 Inocybe species, the species-richest and most recorded mycorrhizal genus. Inocybe species are already known to associate with several orchid species, and have been reported to support seed germination and seedling development in C. Iongifolia and Epipactis atrorubens (Bidartondo \& Read 2008) as well as to colonize C. damasonium, E. atrorubens, and $E$. dunensis adult plant roots in Germany (Bidartondo et al. 2004, Bidartondo \& Read 2008). The two analysed Cephalanthera species have been previously found to associate with several other fungi detected in our sporophore surveys, such as species of Cortinarius, Hebeloma, and Russula simultaneously ectomycorrhizal with neighbouring trees (Bidartondo et al. 2004, Bidartondo \& Read 2008, Liebel et al. 2010).

In spite of the diversity of macromycetes uncovered in the investigated sites, no direct evidence of mycorrhizal associations between above ground fungal species and analysed orchids was found using molecular below ground methods. None of the 268 macrofungal taxa, growing on the various substrates in the vicinity of the orchids, was found to colonize the roots of the 99 orchid samples molecularly analysed.

The eight analysed orchid species were found to associate with a specific range of fungi selected from a strikingly large variety of potential partners. We propose an intriguing scenario where orchid plants are engaged in a complex network of relationships with co-occurring fungi, that is not limited to the restricted number of fungal taxa actually associating with their roots. While we have no direct evidence that orchids interact with fungi that were not identified from orchid roots, the results of our statistical analysis support this possibility. Statistical analyses indicated a correlation between macrofungal diversity and orchid community variation, regardless of the effect of the environmental and spatial factors of the investigated sites. Surprisingly, macrofungi not 


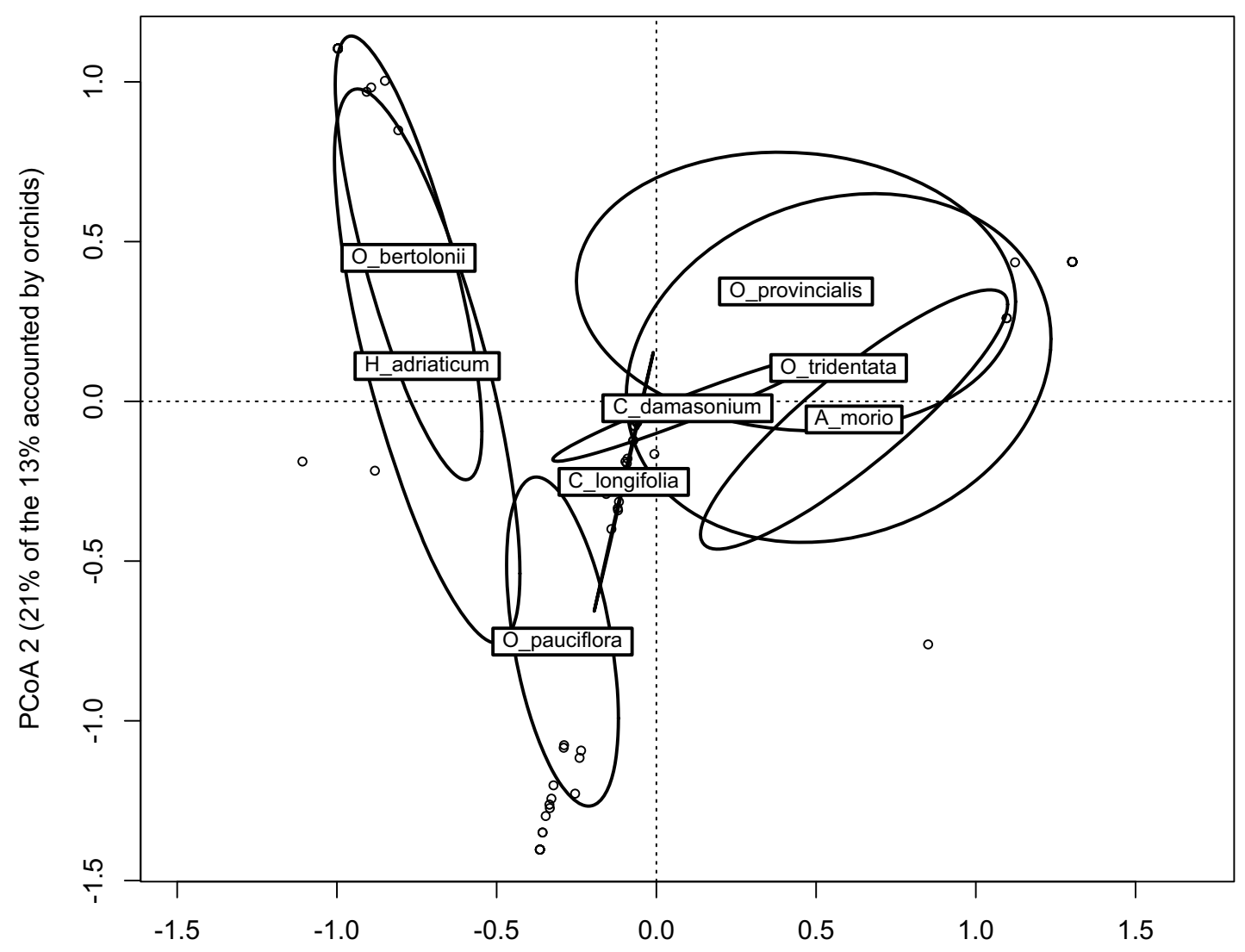

PCoA 1 (53\% of the $13 \%$ accounted by orchids)

Fig. 5. PCoA ordination of the fungal community molecularly detected in orchid roots. Dots are individual root samples while the ellipsoid represent the $95 \%$ confidence limits around each orchid species centroid.

directly detected in the roots explained about $7 \%$ of total orchid community variation. This might be explained by mycorrhizal symbionts varying through stages in the orchid life-cycle, from seed germination to adulthood (Bidartondo \& Read 2008, Jacquemyn et al. 2011, Těšitelová et al. 2015), or as well by the different seasons over the collecting period (Oja et al. 2015, Ercole et al. 2015). The fungal species pool in the soil at a site may therefore play a fundamental role in determining orchid diversity and distribution, providing a source of mycorrhizal partners that form associations with the host plants over time. Second, although factors governing species composition of fungal communities are still poorly understood, it is recognized that combinations of fungal species can be either positively or negatively associated (Jumpponen et al. 2004), perhaps indicating fungus-fungus reciprocal influence that can lead to a certain community structure. So, the occurrence of particular fungi, such as competitors for the same carbon source or fungal parasites, may influence the presence and abundance of orchid mycobionts in natural environments, thus indirectly affecting orchid distribution (Rasmussen et al. 2015). An inability of some orchid mycorrhizal fungi to compete with other fungal species under certain field conditions may be a crucial limiting factor for the establishment of orchid-fungus associations in nature (McCormick et al. 2016, Rock-Blake et al. 2017). Third, soil fungi that do not usually associate with orchids may accidentally have a direct influence on orchid growth and distribution. For instance, they may stimulate orchid seed germination by producing ethylene that is known to increase germination in vitro (Rasmussen 1995). Finally, in a recently recognized plant-fungus symbiosis between jarrah (Eucalyptus marginata) and the basidiomycete Austroboletus occidentalis, the plant accesses fungus derived enhanced nutrients from the rhizosphere, in the absence of fungal root colonization (Kariman et al. 2014). It is tempting to speculate whether fungal mycelia that do not penetrate orchid roots may have beneficial effects for orchid growth and nutrition, as recent studies emphasize the role of fungi in soil biogenic weathering processes, such as mineral dissolution, increasing nutrient availability for plants in several ecosystems (Finlay et al. 2009).

\section{Mycorrhizal associations}

Molecular results showed that a wide range of basidiomycetes and ascomycetes associated with the analysed orchid species, rhizoctonioid fungi dominating the orchid root fungal community, a result consistent with microscopical observations on both root sections and isolated mycelia. Among rhizoctonioid partners, the orchids primarily associated with members of Ceratobasidiaceae, and to a lesser extent Tulasnellaceae, while Sebacina sequences were only amplified from a few Cephalanthera samples. Fungi belonging to Ceratobasidiaceae, Tulasnellaceae, and Sebacinales have been shown to represent the majority of both terrestrial and epiphytic green orchids mycobionts (Taylor et al. 2002, McCormick et al. 2004, McCormick 
\& Jacquemyn 2014) and their partnership in orchid mycorrhizas is considered ancestral (Rasmussen 2002, Dearnaley 2007). Phylogenetic analysis showed that the studied orchids associated with several related taxa within Ceratobasidiaceae and Tulasnellaceae, thus indicating low mycorrhizal specificity. Different Rhizoctonia endophytes colonized roots of the orchid species both within and between sites. Nonetheless, co-occurring orchid species showed a preference for different Rhizoctonia partners. Segregation of associated fungal communities may contribute to the coexistence of Mediterranean orchid species by reducing competition. Based on the analysis of mycorrhizal fungi in 56 individuals from nine different orchid species co-occurring in $25 \times 25$ m Mediterranean grassland plots in southern Italy, Jacquemyn et al. (2014) demonstrated that coexisting orchid taxa were associated with distinct mycorrhizal communities, and suggested niche partitioning as a crucial mechanism affecting the coexistence. Similarly, Waterman et al. (2011) demonstrated that shifts in fungal partners are important for the coexistence of orchid species by showing that each of the six analysed coexisting orchid lineages in Coryciinae had a strong preference for one of the six detected clades of mycorrhizal fungi, that probably allowed coexisting orchid taxa to access different source of nitrogen. The ability of different genera of orchid mycorrhizal fungi, co-occurring in a resource-limited environment, to utilize distinct nutrient sources, was also shown by Nurfadilah et al. (2013), who suggested that fungal differentiation between niches may regulate the coexistence of associated orchid species, and so be a driver for orchid distribution and rarity.

A low level of specificity in the relationship between the orchids and fungi analysed was confirmed by the large variety of ascomycetes and non-Rhizoctonia basidiomycetes detected in the root samples, sometimes replacing the Rhizoctonia partner or co-occurring in the roots of the same plant. Multiple associations and low specificity have been previously shown to characterize mycorrhizal relationships in several Mediterranean orchid species (Jacquemyn et al. 2011, 2014, Girlanda et al. 2011). However, further physiological studies are needed to clarify the roles of associated fungal species detected in the investigated orchid taxa. It is actually possible that some fungi colonizing the orchid roots, especially non-Rhizoctonia species represented by few sequences, do not establish any trophic relationship with the host and just occur as benign endophytes. Nonetheless, the high diversity of orchid mycorrhizal fungi reported here indicates the complexity of relationships between orchids and fungi in nature.

\section{CONCLUSIONS}

This study is the first to examine explicitly both the diversity of mycorrhizal associations involving orchids in a natural environments and the diversity and structure of the fungal community that formed aboveground sexual structures where orchid species grew. This work shows that fungal communities are related to orchid distribution in Mediterranean meadow and forest habitats. The highly diverse macrofungal communities characterising the study sites contribute to the orchid community variation without colonizing the analysed plant roots. Molecular analysis of the orchid root endophytes reveals low mycorrhizal specificity and segregation of associated fungal communities in the studied species, which may contribute to Mediterranean orchids coexistence in natural environments.

Further analyses of the whole fungal communities characterizing orchid growing sites in different environments, based on novel high-throughput sequencing methods, may be expected to provide important information on the complex networks of interactions between orchids and fungi.

\section{ACKNOWLEDGEMENTS}

The experimental work was performed at University of Siena as part of the PhD project of L.P., who also acknowledges CAS $153211 \mathrm{KYSB} 20160029$ for supporting his research at Chinese Academy of Sciences.

\section{REFERENCES}

Arnolds E (1981) Ecology and coenology of macrofungi in grasslands and moist heathlands in Drenthe, The Netherlands. Part 1. Introduction and synecology. Biblioteca Mycologica 83: 1-410.

Bidartondo MI, Read DJ (2008) Fungal specificity bottlenecks during orchid germination and development. Molecular Ecology 17: 3707-3716.

Bidartondo MI, Burghardt B, Gebauer G, Bruns TD, Read DJ (2004) Changing partners in the dark: isotopic and molecular evidence of ectomycorrhizal liaisons between forest orchids and trees. Proceedings of the Royal Society of London, B 271: 1799-1806.

Borcard D, Legendre P (2002) All-scale spatial analysis of ecological data by means of principal coordinates of neighbour matrices. Ecological Modelling 153: 51-68.

Davis BJ, Phillips RD, Wright M, Linde CC, Dixon KW (2015) Continent-wide distribution in mycorrhizal fungi: implications for the biogeography of specialized orchids. Annals of Botany 116: 413-421.

Dearnaley JDW (2007) Further advances in orchid mycorrhizal research. Mycorrhiza 17: 475-486.

Doyle JJ, Doyle JL (1990) Isolation of plant DNA from fresh tissues. Focus 12: 13-15.

Dray S, Legendre P, Peres-Neto PR (2006) Spatial modelling: a comprehensive framework for principal coordinate analysis of neighbour matrices (PCNM). Ecological Modelling 196: 483-493.

Ercole E, Adamo M, Rodda M, Gebauer G, Girlanda M, Perotto S (2015) Temporal variation in mycorrhizal diversity and carbon and nitrogen stable isotope abundance in the wintergreen meadow orchid Anacamptis morio. New Phytologist 205: 1308-1319.

Felsenstein J (1985) Confidence limits on phylogenies: an approach using the bootstrap. Evolution 39: 783-791.

Finlay RD, Wallander H, Smits M, Holmström S, van Hees PAW, Lian $B$, Rosling A (2009) The role of fungi in biogenic weathering in boreal forest soil. Fungal Biology Reviews 23: 101-106.

Gardes M, Bruns TD (1993) ITS primers with enhanced specificity for basidiomycetes - applications to the identification of mycorrhizae and rusts. Molecular Ecology 2: 113-118.

Girlanda M, Segreto R, Cafasso D, et al. (2011) Photosynthetic Mediterranean meadow orchids feature partial mycoheterotrophy 
and specific mycorrhizal associations. American Journal of Botany 98: 1148-1163.

Gotelli NJ, Ellison AM (2004) A Primer of Ecological Statistics. Sunderland, MA: Sinauer Associates.

Jacquemyn H, Brys R, Cammue BPA, Honnay O, Lievens B (2011) Mycorrhizal associations and reproductive isolation in three closely related Orchis species. Annals of Botany 107: 347-356.

Jacquemyn H, Brys R, Honnay O, Roldán-Ruiz I, Lievens B, Wiegand T (2012) Non-random spatial structuring of orchids in a hybrid zone of three Orchis species. New Phytologist 193: 454-464.

Jacquemyn H, Brys R, Merckx VSFT, Waud M, Lievens B, Wiegand T (2014) Co-existing orchid species have distinct mycorrhizal communities and display strong spatial segregation. New Phytologist 202: 616-627.

Jumpponen A, Claridge AW, Trappe JM, Lebel T, Claridge DL (2004) Ecological relationships among hypogeous fungi and trees: inferences from association analysis integrated with habitat modeling. Mycologia 96: 510-525.

Kariman K, Barker SJ, Jost R, Finnegan PM, Tibbett M (2014) A novel plant-fungus symbiosis benefits the host without forming mycorrhizal structures. New Phytologist 201: 1413-1422.

Kosaka N, Kawahara T, Takahashi H (2014) Vegetation influencing the establishment and growth of the endangered Japanese orchid, Cypripedium macranthos var. rebunense. Ecological Research 29: 1003-1023.

Larkin MA, Blackshields G, Brown NP, Chenna R, McGettigan PA, McWilliam H, Valentin F, et al. (2007) Clustal W and Clustal $X$ version 2.0. Bioinformatics 23: 2947-2948.

Leake JR (1994) The biology of mycoheterotrophic ('saprophytic') plants. [Tansley Review No. 69.] New Phytologist 127: 171-216.

Legendre P, Gallagher ED (2001) Ecologically meaningful transformations for ordination of species data. Oecologia 129: 271-280.

Legendre P, Legendre L (1998) Numerical Ecology. Amsterdam: Elsevier.

Liebel HT, Bidartondo MI, Preiss K, et al. (2010) C and N stable isotope signatures reveal constraints to nutritional modes in orchids from the Mediterranean and Macaronesia. American Journal of Botany 97: 903-912.

McCormick MK, Jacquemyn $\mathrm{H}$ (2014) What constrains the distribution of orchid populations? New Phytologist 202: 392-400.

McCormick MK, Whigham DF, O'Neill JP (2004) Mycorrhizal diversity in photosynthetic terrestrial orchids. New Phytologist 163: 425438.

McCormick MK, Whigham DF, O'Neill JP, Becker JJ, Werner S, Rasmussen HN, Bruns TD, Taylor DL (2009) Abundance and distribution of Corallorhiza odonthoriza reflect variations in climate and ectomycorrhizae. Ecological Monographs 79: 619635.

McCormick MK, Taylor DL, Juhaszova K, Burnett RKJR, Whigham DF, O'Neill JP (2012) Limitations on orchid recruitment: not a simple picture. Molecular Ecology 21: 1511-1523.

McCormick MK, Taylor DL, Whigham DF, Burnett RKJR (2016) Germination patterns in three terrestrial orchids relate to abundance of mycorrhizal fungi. Journal of Ecology 104: 744754.

Nurfadilah S, Swarts ND, Dixon KW, Lambers H, Merritt DJ (2013) Variation in nutrient-acquisition patterns by mycorrhizal fungi of rare and common orchid explains diversification in a global biodiversity hotspot. Annals of Botany 111: 1233-1241.
Oja J, Kohout P, Tedersoo L, Kull T, Kõljalg U (2015) Temporal patterns of orchid mycorrhizal fungi in meadows and forests as revealed by 454 pyrosequencing. New Phytologist 205: 1608-1618.

Oksanen J, Guillaume Blanchet F, Kindt R, Legendre P, Minchin PR, O'Hara RB, et al. (2012) Vegan: Community ecology package.

Pecoraro L, Girlanda M, Kull T, Perini C, Perotto S (2012a) Analysis of fungal diversity in Orchis tridentata Scopoli. Central European Journal of Biology 7: 850-857.

Pecoraro L, Girlanda M, Kull T, Perini C, Perotto S (2012b) Molecular identification of root fungal associates in Orchis pauciflora Tenore. Plant Biosystems 146: 985-991.

Pecoraro L, Girlanda M, Kull T, Perini C, Perotto S (2013) Fungi from the roots of the terrestrial photosynthetic orchid Himantoglossum adriaticum. Plant Ecology and Evolution 146: 145-152.

Pecoraro L, Girlanda M, Liu ZJ, Huang LQ, Perotto S (2015) Molecular analysis of fungi associated with the Mediterranean orchid Ophrys bertolonii Mor. Annals of Microbiology 65: 2001-2007.

Pecoraro L, Huang LQ, Caruso T, Perotto S, Girlanda M, Cai L, Liu ZJ (2017) Fungal diversity and specificity in Cephalanthera damasonium and C. longifolia (Orchidaceae) mycorrhizas. Journal of Systematics and Evolution 55: 158-169.

R Core Team (2015) R: A language and environment for statistical computing. R Foundation for Statistical Computing, Vienna.

Rasmussen HN (1995) Terrestrial orchids: from seed to mycotrophic plant. Cambridge: Cambridge University Press.

Rasmussen HN (2002) Recent developments in the study of orchid mycorrhiza. Plant and Soil 244: 149-163.

Rasmussen HN, Dixon KW, Jersáková J, Těšitelová T (2015) Germination and seedling establishment in orchids: a complex of requirements. Annals of Botany 116: 391-402.

Rock-Blake R, McCormick MK, Brooks HEA, Jones CS, Whigham DF (2017) Symbiont abundance can affect host plant population dynamics. American Journal of Botany 104: 72-82.

Tamura K, Peterson D, Peterson N, Stecher G, Nei M, Kumar S (2011) MEGA5: Molecular Evolutionary Genetics Analysis using maximum likelihood, evolutionary distance, and maximum parsimony methods. Molecular Biology and Evolution 28: 27312739.

Taylor DL, Bruns TD, Leake JR, Read DJ (2002) Mycorrhizal specificity and function in myco-heterotrophic plants. In: Mycorrhizal Ecology (Van der Heijden MJA, Sanders IR, eds): 375-413. Berlin: Springer-Verlag.

Těšitelová T, Těšitel J, Jersáková J, Rihová G, Selosse MA (2012) Symbiotic germination capability of four Epipactis species (Orchidaceae) is broader than expected from adult ecology. American Journal of Botany 99: 1020-1032.

Těšitelová T, Kotilínek M, Jersáková J, et al. (2015) Two widespread green Neottia species (Orchidaceae) show mycorrhizal preference for Sebacinales in various habitats and ontogenetic stages. Molecular Ecology 24: 1122-1134.

Vogt KA, Bloomfield J, Ammirati JF, Ammirati SR (1992) Sporocarp production by basidiomycetes, with emphasis on forest ecosystems. In: The Fungal Community: its organization and role in the ecosystem (Carroll GC, Wicklow DT, eds): 563-581. New York: Marcel Dekker.

Waterman RJ, Bidartondo MI, Stofberg J, Combs JK, Gebauer G, et al. (2011) The effects of above- and belowground mutualisms on orchid speciation and coexistence. American Naturalist 177: E54-E68. 


\section{Supporting Information}

Additional Supporting Information may be found in the online version of this article:

Table S1. Detailed description of biotic and abiotic factors characterizing each investigated plot $(p)$, including list of orchid and macrofungal species, environmental and geographical factors. Number of sporocarps for each macrofungal taxon is reported.

Table S2. Overview of all root-associated fungi molecularly detected in the eight analysed orchid species.
Table S3. BLAST search closest matches of fungal ITS-DNA sequences amplified from $A$. morio and $O$. provincialis roots collected in "Monte Cetona" (samples MC1-MC4) and "Monte Rotondo" (samples MR1-MR24). Sample GenBank accession codes, accession codes for the closest GenBank matches, sequence identity, and overlap of each match are reported.

Fig. S1. Principal Component Analysis (PCA) of environmental variables (habitat, tree, shrub, herb, and lichen-moss cover $\%$, litter cover $\%$, rock cover $\%$, stone cover $\%$, micro-topography, elevation above sea level, slope $\%$, and orientation). 